\title{
Reconstruction of a large nasal defect using a folded forehead flap: a case report
}

\author{
Minkyoung Jeong, \\ Dongkeun Jun ${ }^{1}$, Jeenam Kim', \\ Hyungon Choi ${ }^{1}$, Donghyeok Shin ${ }^{1}$, \\ Jaehoon $\mathrm{Cho}^{2}$, Youngchang $\mathrm{Lim}^{2}$, \\ Myungchul Lee ${ }^{1}$ \\ Departments of ${ }^{1}$ Plastic and \\ Reconstructive Surgery and \\ ${ }^{2}$ Otorhinolaryngology, Konkuk University \\ Medical Center, Seoul, Korea
}

\begin{abstract}
Forehead flaps are widely used to reconstruct nasal defects. The authors report a case wherein a folded forehead flap was used to reconstruct a large nasal defect after wide excision of squamous cell carcinoma. A 65-year-old man was diagnosed with squamous cell carcinoma by a punch biopsy conducted at the dermatology department, and the mass was located in the left nasal vestibule. A forehead flap was planned to cover the full-thickness defect that occurred after wide excision. A flap with an extended transverse skin paddle was designed; thereafter, the distant part of the flap was folded up to the nasal lining inside the nose. The interpolation flap was properly maintained for 3 weeks, and flap division was performed. The reconstructed nose exhibited symmetry during a 5-month observation period. A folded forehead flap is a surgical option when considerable nasal restoration, including soft tissue and the internal lining, is necessary.
\end{abstract}

Keywords Nose / Reconstruction / Forehead / Flap / Case reports

\section{INTRODUCTION}

The aesthetic importance of the nose underscores the need to restore the patient's appearance by reconstructing defects that occur due to trauma, tumor, or infection [1-3]. Various nasal reconstruction methods have been introduced, including the nasolabial flap, composite grafting, the forehead flap, and the radial forearm free flap [4]. However, the range of surgical options can be limited when all layers of the nasal tissue require restoration. In such cases, cautious preoperative planning is necessary.

Forehead flaps are considered to be the best option for reconstruction of the nose [5]. Usually, a forehead flap proceeds in two stages. First, it is harvested from the forehead, including the frontalis muscle and subcutaneous fat, and then it is inset into the recipient site. After approximately 3 weeks, flap division is performed to

Received: Oct 7, 2021 Revised: Nov 19, 2021 Accepted: Nov 22, 2021 Correspondence: Myungchul Lee Department of Plastic and Reconstructive Surgery, Konkuk University Medical Center, 120-1 Neungdong-ro, Gwangjingu, Seoul 05030, Korea

Tel: +82-2-2030-7639, Fax: +82-2-2030-7733, E-mail: 20150136@kuh.ac.kr

Copyright @ 2022 The Korean Society for Aesthetic Plastic Surgery.

This is an Open Access article distributed under the terms of the Creative Commons Attribution Non-Commercial License (https://creativecommons.org/licenses/by-nc/4.0/) which permits unrestricted non-commercial use, distribution, and reproduction in any medium, provided the original work is properly cited. www.e-aaps.org separate the pedicle [6,7]. The main blood supply of the forehead flap is the supratrochlear artery, which branches from the ophthalmic artery $[8,9]$. In some cases, minor revision surgery is required to restore the natural contour and symmetry [10]. In this study, we report a folded forehead flap procedure following wide excision of squamous cell carcinoma on the nasal vestibule.

\section{CASE REPORT}

A 65-year-old man visited the department of plastic and reconstructive surgery for a lesion on the left nasal vestibule that developed 1 month previously. The lesion measured $2 \mathrm{~cm}$ in size, was slightly yellowish, and had an irregular, ulcer-like shape (Fig. 1A). The diagnosis of squamous cell carcinoma was confirmed by a punch biopsy, and a computed tomography scan showed invasion of the left nasal vestibule and columella. Wide excision and reconstruction using a forehead flap were planned. Under general anesthesia, wide excision was performed with surgical margins of $6 \mathrm{~mm}$ cranially and on both sides, and $10 \mathrm{~mm}$ caudally with full thickness, including the left alar skin, cartilage, and mucosa (Fig. 1B). A frozen biopsy examination showed that the cranial extent of the tumor was close to the margin, and additional excision was performed with a safety margin of $5 \mathrm{~mm}$. A further frozen biopsy examination confirmed that the margin was free. The defect size was $3 \times 3 \mathrm{~cm}$; this wide-ranging full-thickness defect included the nasal tip, colu- 

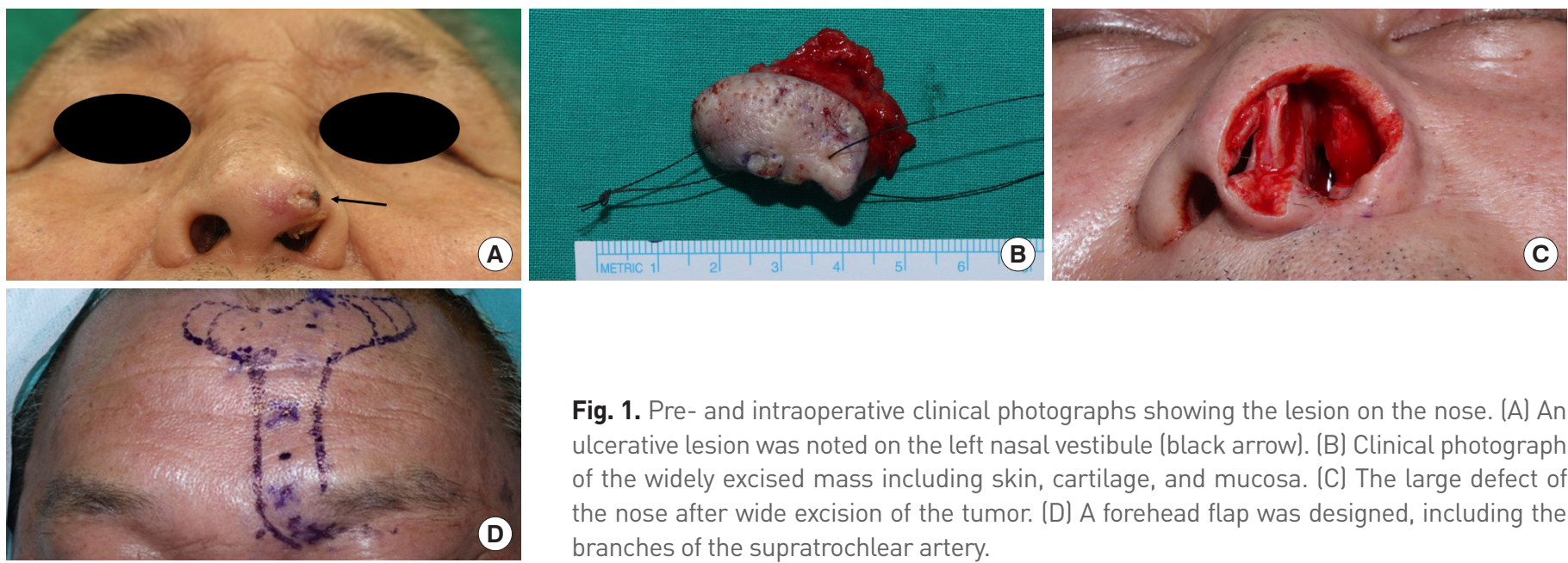

Fig. 1. Pre- and intraoperative clinical photographs showing the lesion on the nose. (A) An ulcerative lesion was noted on the left nasal vestibule (black arrow). (B) Clinical photograph of the widely excised mass including skin, cartilage, and mucosa. (C) The large defect of the nose after wide excision of the tumor. (D) A forehead flap was designed, including the branches of the supratrochlear artery.
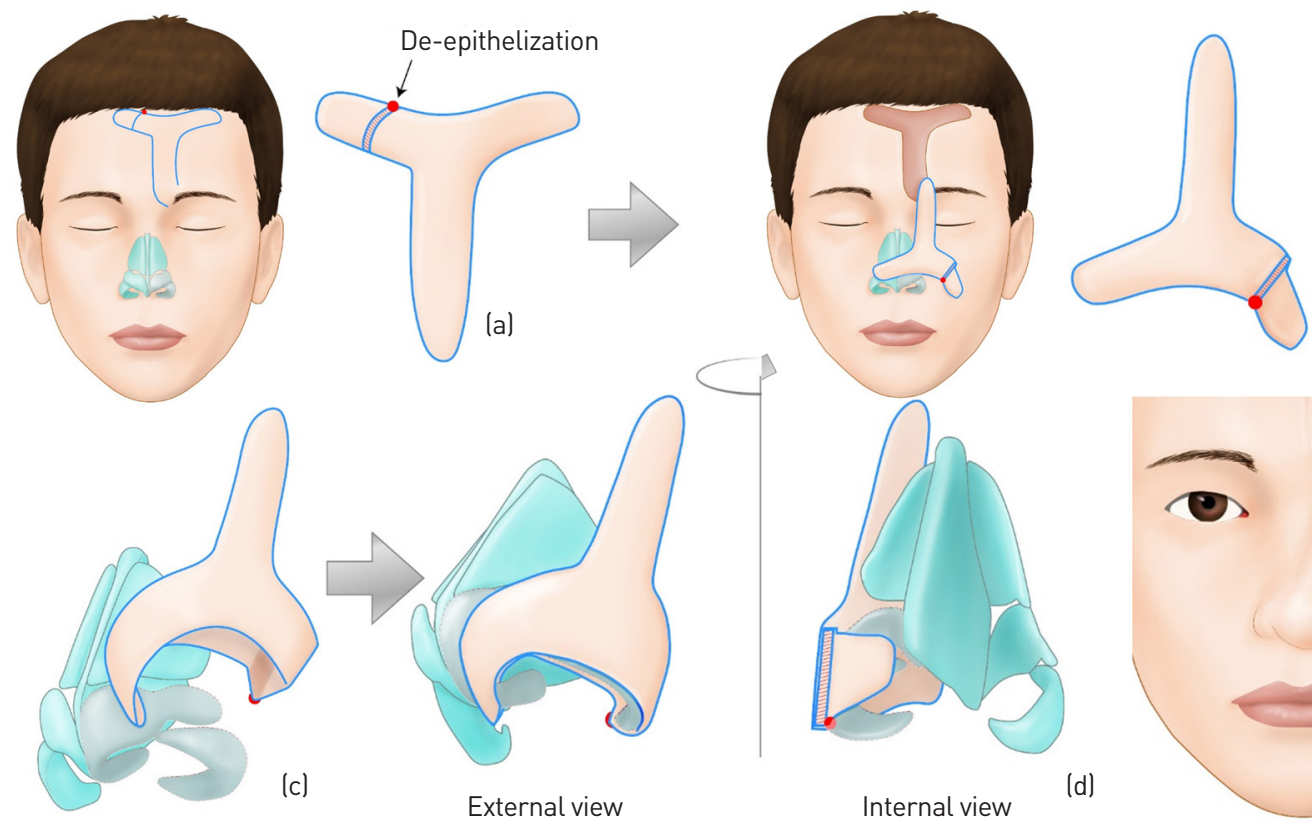

(b)

(c)
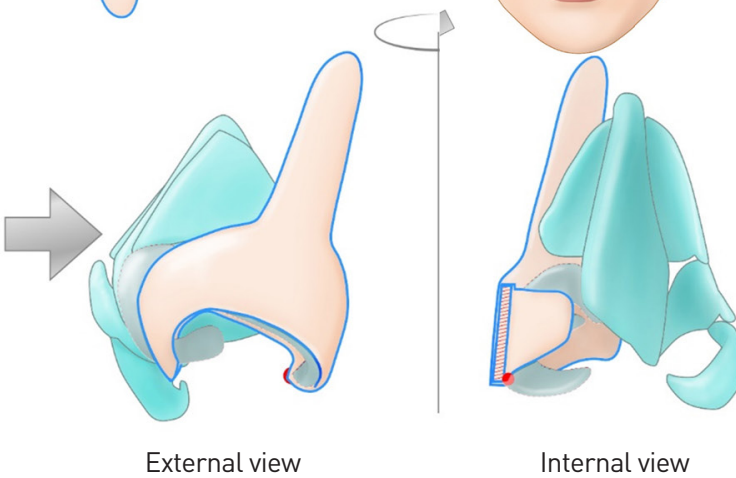

Internal view

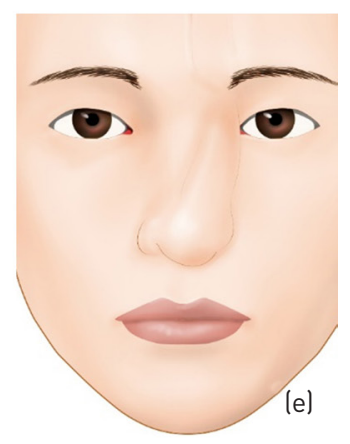

Fig. 2. Schematic illustration of the forehead flap procedure. (a) A flap with an extended transverse skin paddle was designed and part of it underwent de-epithelization. (b) The flap was transposed and folded to reconstruct the alar rim and inner lining. (c) Flap in-setting with regard to both the columella and alar area. (d, e) Simultaneous reconstruction of the outer skin and inner mucosal lining, with folding of the forehead flap.

mella, and nasal ala (Fig. 1C). The supratrochlear artery was probed using Doppler tracing. A forehead flap with an extended transverse skin paddle was designed that would be suitable for folding (Fig. 1D). After flap elevation, transposition was performed toward the nose, and the flap was folded inward to be inset in the area of the defect (Fig. 2). The elevated flap was inset for nasal reconstruction, and most of the raw surface on the forehead was repaired using primary closure. Nonetheless, a split-thickness skin graft $(1 \times 1 \mathrm{~cm})$ was necessary on the three points where the bilateral forehead skin and scalp tissue adjoined.

Three weeks after surgery, the pedicle was separated by flap divi- sion under local anesthesia. The bridging pedicle was tightened lightly during the division procedure, and the flap color was maintained without vascular compromise. The pedicle was trimmed so that an adequate approximation would be possible between the flap and the cephalic margin of the nasal defect. The medial border of the eyebrow where the supratrochlear artery originated was examined carefully. A triangular flap $(1.5 \times 1 \mathrm{~cm})$ was designed and returned, considering symmetry between the bilateral eyebrows. The rest of the bridging tissue was discarded.

Radiation therapy was performed for 1 month after the postoperative wound healing, and a nasal stent was kept in place for 2 

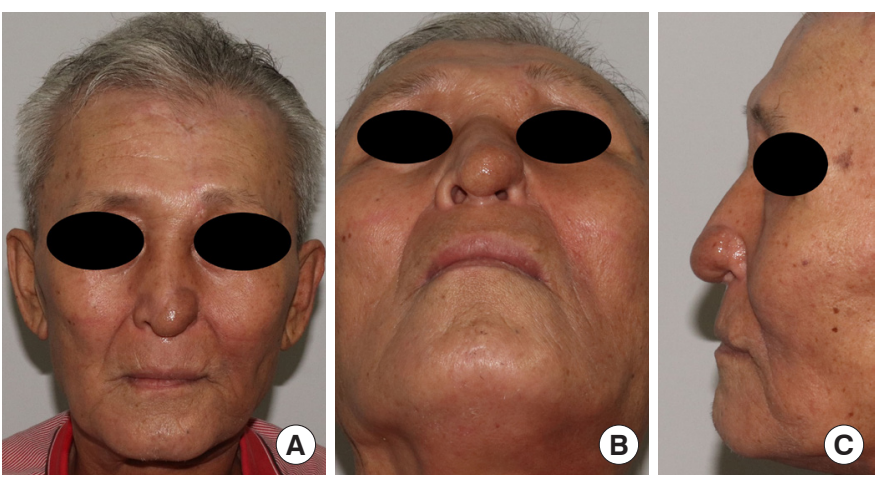

Fig. 3. Postoperative status ( 4 months after flap division). (A) Postoperative photograph showing that the forehead flap had healed well. Worm's eye view $(\mathrm{B})$ and lateral view $(\mathrm{C})$ in postoperative photographs.

months to maintain the nasal airway. The patient recovered well without remarkable events and showed symmetric nasal alae during 5 months of follow-up, including the period of radiation therapy (Fig. 3). Furthermore, the nasal airway was maintained well in the course of respiration. In postoperative nasal endoscopy, the junction between the forehead flap and nasal mucosa had healed, and the nasal passage showed an intact inner membrane (Fig. 4).

\section{DISCUSSION}

Due to its texture and color, forehead flaps are considered to be suitable for the reconstruction of nasal defects $[2,4]$. It is known that separate flaps (e.g., mucosal flaps or free flaps) are needed to simultaneously reconstruct the nasal lining inside the nose when the entire layer of the nose is missing [11-13]. However, reconstruction of the mucosal lining with a mucosal flap is inconvenient in terms of the donor site, and free flaps have the limitation of resulting in a bulky nose $[13,14]$.

In addition, existing designs of folded forehead flaps are difficult to use for simultaneous reconstruction of the entire alar rim, columella, and nasal lining $[12,14]$. Therefore, a long flap in the transverse direction was designed and properly folded to reconstruct the entire alar rim, columella, and nasal lining. Although a forehead flap with a supratrochlear pedicle may be easy to extend towards the scalp in the vertical direction, it may not provide sufficient blood flow in the transverse direction [9]. Therefore, Doppler tracing was carefully performed before and during surgery, and the blood flow of the distal part of the transverse flap was checked during the procedure. This procedure was carried out once it was ascertained that there was a communicating branch beyond the midline in the transverse direction of the supratrochlear artery anatomy, as well as a branch between the supratrochlear artery and supraorbital artery. Therefore, leaving those structures intact to secure blood flow would not pose an obstacle for flap elevation [9].

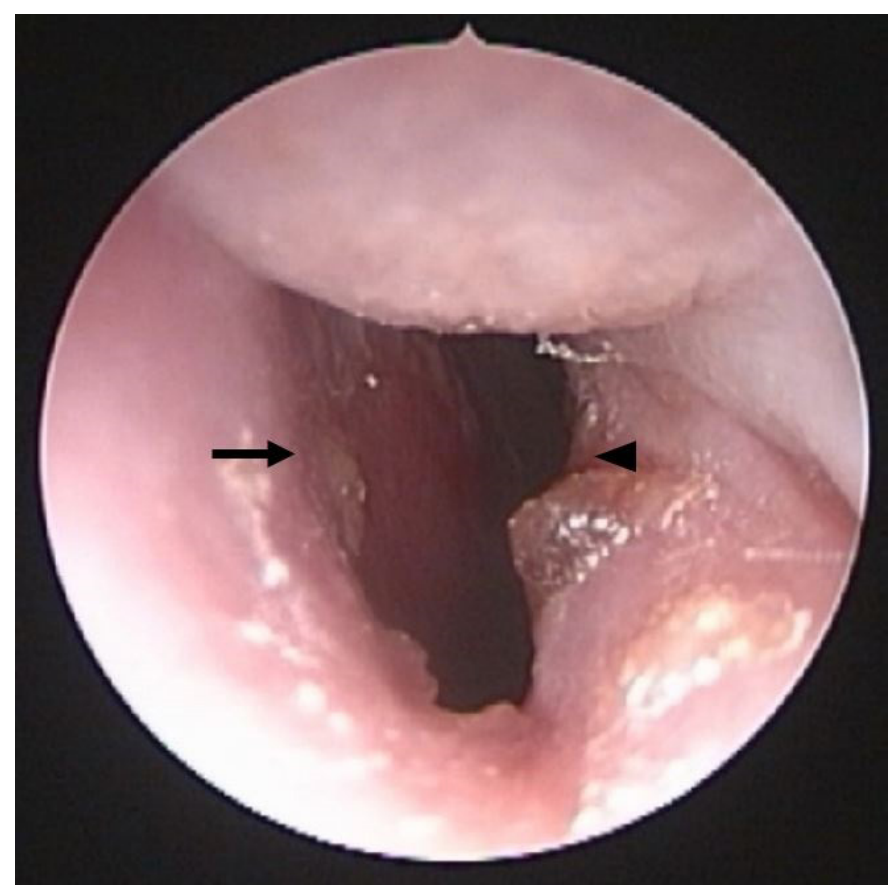

Fig. 4. Postoperative nasal endoscopy image. The forehead flap was maintained well at a 7-month follow-up, and the junction between the flap and nasal septal mucosa had healed without complications (black arrow). The lateral wall showed an intact passage where the flap had been folded (black arrowhead).

During the wide excision process, a cartilage graft may be required to maintain the shape of the nostrils, but in this case, the skin and soft tissue were folded together like a sandwich and the thickness of the flap was maintained. Thus, the shape of the alar rim was formed, and the defect was reconstructed only by flap in-setting without a separate cartilage graft.

Three weeks after surgery, flap division under local anesthesia was performed. Studies in the literature recommend that, at 3 weeks after surgery, if the flap color becomes purple after pressing the pedicle lightly, it is appropriate to wait another week and perform the division 4 weeks after surgery $[8,11]$. In this case, when the flap was lightly tightened at week 3 , the color was well maintained, so the division was carried out at week 3. At a 5-month follow-up, including radiation therapy for 1 month after division, there were no wound problems and the flap remained intact.

With regard to the limitations of this study, the reconstructed nasal ala and lateral wall showed some degree of bulkiness, and an additional debulking procedure would lead to a more natural contour. Nonetheless, the patient was satisfied with the functional outcomes. Furthermore, the reconstructed nostril was somewhat smaller than that on the right side, and a cartilage graft procedure could provide support for the nasal framework. To summarize, we report a case of composite reconstruction of a large nasal defect with a folded forehead flap. 


\section{NOTES}

\section{Conflict of interest}

No potential conflict of interest relevant to this article was reported.

\section{Ethical approval}

The protocol of this study was approved for exemption by the Institutional Review Board (IRB exemption No. KUMC 2021-04-055).

\section{Patient consent}

The patient provided written informed consent for the publication and the use of his images.

\section{ORICD}

Minkyoung Jeong

Dongkeun Jun

Jeenam Kim

Hyungon Choi

Donghyeok Shin

Jaehoon Cho

Myungchul Lee

https://orcid.org/0000-0002-8250-0388

https://orcid.org/0000-0001-9017-9929

https://orcid.org/0000-0002-4080-6135

https://orcid.org/0000-0002-3816-1286

https://orcid.org/0000-0002-8450-4411

https://orcid.org/0000-0002-2243-7428

https://orcid.org/0000-0002-9721-0092

\section{REFERENCES}

1. Lim J, Oh J, Eun S. Flap reconstruction of soft tissue defect after resecting a huge hemangioma of the nose. Arch Craniofac Surg 2020; 21:69-72.

2. Hammer D, Williams F, Kim R. Paramedian forehead flap. Atlas Oral Maxillofac Surg Clin North Am 2020;28:23-8.

3. Park YJ, Kwon GH, Kim JO, et al. Reconstruction of nasal ala and tip following skin cancer resection. Arch Craniofac Surg 2019;20:382-7.

4. Chioaru B, Dragu E, Lascar I. Nose defects reconstruction with forehead flap: case report. Maedica (Bucur) 2014;9:76-8.

5. Rudolph MA, Walker NJ, Rebowe RE, et al. Broadening applications and insights into the cross-paramedian forehead flap over a 19-year period. J Plast Reconstr Aesthet Surg 2019;72:763-70.

6. Ni HC, Wang JC, Sun JM, et al. Expanded paramedian forehead flaps for nasal defects: beyond aesthetic subunits. Ann Plast Surg 2018;81 (6S Suppl 1):S54-8.

7. Shokri T, Kadakia S, Saman M, et al. The paramedian forehead flap for nasal reconstruction: from antiquity to present. J Craniofac Surg 2019;30:330-3.

8. Agorgianitis L, Panagouli E, Tsakotos G, et al. The supratrochlear artery revisited: an anatomic review in favor of modern cosmetic applications in the area. Cureus 2020;12:e7141.

9. Angobaldo J, Marks M. Refinements in nasal reconstruction: the crossparamedian forehead flap. Plast Reconstr Surg 2009;123:87-93.

10. Menick FJ. Nasal reconstruction. Plast Reconstr Surg 2010;125:138e150 e.

11. Correa BJ, Weathers WM, Wolfswinkel EM, et al. The forehead flap: the gold standard of nasal soft tissue reconstruction. Semin Plast Surg 2013;27:96-103.

12. Alagoz MS, Isken T, Sen C, et al. Three-dimensional nasal reconstruction using a prefabricated forehead flap: case report. Aesthetic Plast Surg 2008;32:166-71.

13. Menick FJ. A 10-year experience in nasal reconstruction with the threestage forehead flap. Plast Reconstr Surg 2002;109:1839-55.

14. Zelken JA, Chang CS, Reddy SK, et al. Double forehead flap reconstruction of composite nasal defects. J Plast Reconstr Aesthet Surg 2016;69:1280-4. 